\title{
ABHANDLUNGEN
}

\section{Rationale Rekonstruktion als Methode politischer Theorie zwischen Gesellschaftskritik und empirischer Politikwissenschaft}

\author{
Daniel Gaus
}

\section{Rational Reconstruction as Method of Political Theory between Critique and Empirical Political Science}

Abstract: Current political theory applies the concept of reconstruction almost exclusively to methods of critique. Given this focus it is often overlooked that the concept of reconstruction also has important methodological implications regarding the empirical analysis of politics. This imbalance particularly applies to how Jürgen Habermas' discourse theory of democracy has been perceived in political theory. The aim of the article is two-fold. Firstly, it offers an interpretation of discourse theory as a contribution to a "reconstructive sociology of democracy" that goes beyond the mere purpose of critique. Secondly, it seeks to illustrate the added value of Habermas' method of rational reconstruction to the empirical analysis of politics.

Keywords: Methodology, Rational Reconstruction, Political Theory, Habermas, Discourse Theory

Schlagwörter: Methoden, rationale Rekonstruktion, Politische Theorie, Habermas, Diskurstheorie

\section{Einleitung}

In der politischen Theorie ist die Frage nach Gegenstand und Methoden des Fachs ein wiederkehrendes Thema. Immer wieder ist sie Anlass zur Reflexion über das Verhältnis politischer Theorie zu anderen Teilbereichen der Politikwissenschaft einerseits sowie zu benachbarten Fächern, vor allem der Philosophie, andererseits. ${ }^{1}$ Was als Suche nach disziplinärer Identität erscheinen könnte, ist Ausdruck einer Funktion, die die politische Theorie im Kontext der Analyse von Politik erfüllt. Als Brücke oder Vermittlerin zwischen empirisch verfahrender Politikwissenschaft einerseits und Philosophie andererseits ist sie in besonderem Maße Konflikten ausgesetzt, denen ihre Nachbardisziplinen unter Verweis auf ihre jeweilige

1 Ich danke Peter Niesen, Rainer Schmalz-Bruns, Oliver Schmidtke, den Teilnehmern des Kolloquiums Politische Theorie der TU Darmstadt sowie den beiden anonymen Gutachtern für wertvolle Kommentare und Kritik. Die Fertigstellung des vorliegenden Artikels erfolgte während eines Fellowships am Käte Hamburger Kolleg „Politische Kulturen der Weltgesellschaft“ der Universität Duisburg-Essen, für dessen großzügige Unterstützung ich mich bedanke. 
Fachidentität ausweichen können. Indem sie Übersetzungsleistungen in die eine wie in die andere Richtung erbringt, wird die politische Theorie zum Marktplatz, auf dem eingespielte und oft gegensätzliche Denk- und Forschungstraditionen in Philosophie und Politikwissenschaft regelmäßig aufeinander treffen und der Vermittlung harren.

In gegenwärtigen Auseinandersetzungen um die Methoden politischer Theorie spielt der Begriff der Rekonstruktion in der Debatte um das Selbstverständnis politischer Theorie als Gesellschaftskritik eine hervorgehobene Rolle. Hier wird er als Kennzeichnung eines bestimmten Verfahrens der Bestimmung moralischpraktischer Maßstäbe diskutiert, die in der Kritik politischer Verhältnisse zugrundegelegt werden. In normativer Absicht verfahrende Gesellschaftskritik repräsentiert allerdings nur einen, wenngleich einen zweifellos wichtigen Kontext politischer Theorie. Ein zweiter Kontext politischer Theorie lässt sich mit Autoren wie David Easton oder Giovani Sartori als Ausarbeitung einer allgemeinen Theorie der Politik begreifen, deren Modelle und Begriffe der Orientierung empirisch verfahrender Politikwissenschaft dienen können.

Die These, die ich im Folgenden erläutern will, lautet, dass die Bedeutung des Begriffs Rekonstruktion für die Methode politischer Theorie gegenwärtig zu einseitig auf den Kontext der Gesellschaftskritik bezogen wird. Hierbei wird leicht übersehen, dass derselbe Problemzusammenhang, der den Hintergrund der Debatte um rekonstruktive Verfahren der Gesellschaftskritik bildet, wichtige Implikationen für eine Theorie der Politik hat, die nach angemessenen Verfahren der empirischen Analyse politischer Praxis fragt. Diese Einseitigkeit zeigt sich nirgends deutlicher als an der Rezeption, die die rekonstruktiv verfahrende Diskurstheorie des demokratischen Rechtsstaats von Jürgen Habermas (1992) durch die politische Theorie erfahren hat. Sie wird fast ausnahmslos unter dem Gesichtspunkt betrachtet, inwiefern sie zu einer angemessenen Kritik gegenwärtiger politischer Verhältnisse beitragen kann. Im Gegensatz dazu soll gezeigt werden, dass sich die Bedeutung des Begriffs Rekonstruktion im Habermasschen Denken nicht auf den Kontext evaluativ verfahrender Gesellschaftskritik beschränkt, sondern wichtige Implikationen für die empirisch verfahrende Analyse politischer Praxis hat. Jenseits der Idee rekonstruktiver Gesellschaftskritik liegt mit der Habermasschen Theorie auch ein Programm für eine „rekonstruktiv verfahrende Soziologie der Demokratie“ (Habermas 1992, S. 349) vor.

Dazu werde ich in drei Schritten vorgehen. Zunächst schlage ich vor, den Begriff der Rekonstruktion in dem Sinne zu verstehen, wie er von John Dewey 1920 in seiner Forderung nach einer „Reconstruction in Philosophy“ entfaltet worden ist. Dewey erläutert dort den Übergang zur Moderne als Prozess der Rekonstruktion des gesellschaftlichen Bewusstseins welterschließender Praxis (2). Zwar stellt Habermas keinen expliziten Bezug zu Dewey her, seine Theorie lässt sich jedoch als eine Weiterführung von Deweys Gedanken interpretieren. Das erschließt sich allerdings nur, wenn man Habermas' Erläuterungen zum Begriff rationaler Rekonstruktion nicht allein auf den Kontext kritischer Gerechtigkeitstheorie bezieht. In Auseinandersetzung mit Axel Honneths (2011) jüngstem Vorschlag kritischer Gesellschaftsanalyse als „normativer Rekonstruktion“ werde ich deshalb in einem zweiten Schritt argumentieren, dass Habermas' Überlegungen tiefer an- 
setzen (3). Vor diesem Hintergrund lässt sich dann skizzieren, inwiefern die Diskurstheorie des demokratischen Rechtsstaats als eine rationale Rekonstruktion begriffen werden kann, mit der sich auch ein soziologisch-explanativer Geltungsanspruch verbindet. Dieses Verständnis der Habermasschen Theorie impliziert rationale Rekonstruktion als wichtige Ergänzung zu bestehenden empirischen Verfahren, um dem Ziel einer kontextsensiblen Analyse politischer Praxis näher zu kommen (4).

\section{John Deweys „Reconstruction in Philosophy“ (1920)}

Wenn ich im Folgenden ein Denkmotiv Deweys knapp skizzieren möchte, geht es weder darum, Deweys Philosophie in umfassender Weise darzustellen ${ }^{2}$, noch darum, eine direkte Verbindung zwischen Dewey und Habermas zu behaupten. ${ }^{3}$ Vielmehr scheint mir der gedankliche Hintergrund, in dem Dewey den Begriff der Rekonstruktion einführt, mit dem Habermasschen so weit übereinzustimmen, dass er den anschließenden Nachvollzug der Habermasschen Überlegungen zum Rekonstruktionsbegriff erleichtert. Deweys Anliegen in „Reconstruction in Philosophy" ist es, auf den sozialen Entstehungskontext der Konzeption klassischer Philosophie hinzuweisen, um deren (1920) fortdauernde Geltung in Frage zu stellen. Seine These ist, dass es einer Erneuerung der Philosophie bedarf, weil sich im Übergang zur Moderne im Alltagsbewusstsein ein Weltbildwandel vollzogen hat, der den wesentlichen Postulaten der klassischen Philosophie den Boden entzieht. Dieser Weltbildwandel besteht für Dewey vornehmlich darin, die alltägliche welterschließende Praxis mehr und mehr als einen Prozess der kontinuierlichen Rekonstruktion menschlicher Erfahrung wahrzunehmen. Die Konsequenzen dieser alltagspraktischen Transformation für die theoriegeleitete Erzeugung von Wissen herauszuarbeiten - darin besteht meines Erachtens ein Motiv, das Dewey und Habermas teilen.

Dewey zufolge lässt sich die Entstehung der klassischen Philosophie auf eine bestimmte gesellschaftliche Konstellation zurückführen. Demnach ist der Ursprung der Philosophie nicht durch ein reines Erkenntnisinteresse begründet. Vielmehr erfüllte umgekehrt die Konzeption von Theorie als von Praxis getrennt und auf reine Erkenntnis gerichtet eine politische Legitimationsfunktion. Sie diente dazu, die bis dato bestehende Sozialordnung aufrechtzuerhalten. Über die Einführung einer abstrakten und stark systematisierten ,ultra-scientific' Form der Argumentation einerseits sowie über die Trennung zweier Sphären des Seins, der noumenalen und der phänomenalen Welt, andererseits, schirmte sie Dewey zufolge die Geltung der Inhalte des traditionalen mythischen Glaubens ab gegen die Herausforderungen eines zunehmenden, aus dem experimentellen Umgang mit der Natur gewonnenen Profanwissens. Im Lichte dieser Dualität noumenaler und phänomenaler Welt beschränkte sich die Funktion philosophisch erzeugten Wis-

2 Für eine anschauliche Darstellung der Philosophie Deweys vgl. Jörke (2003).

3 Wie Habermas (2002, S. 227-228) selbst bemerkt, gibt es keine solche direkte Verbindung. Zum Verhältnis von Habermas‘ Denken und der pragmatistischen Philosophie vgl. Aboulafia et al. (2002), Larmore (2000) sowie kritisch Joas (1992b). 
sens darauf, eine in ihren Zwecken und möglichen Erscheinungen bereits fixierte Ordnung des Seins aufzudecken. "A world of a limited number of classes, kinds, forms, distinct in quality [...] and arranged in a graded order of superiority and inferiority" (Dewey 1920, S. 54-55).

Dewey zufolge konnte jedoch dieses vormoderne Weltbild dem Druck eines durch naturwissenschaftlichen Fortschritt gewandelten Naturverständnisses schließlich nicht Stand halten. Die Ablösung des geo- durch das heliozentrische Weltbild in der kopernikanischen Wende beschreibt er als eine langsame Bewusstseinstransformation, die mit einem Einstellungswandel hinsichtlich zahlreicher Grundannahmen einherging, welche die Säulen des dualen Weltbildes - und der klassischen Philosophie - ausmachten. Die Annahme einer geschlossenen, fixierten Ordnung der Dinge wurde abgelöst durch die Vorstellung von Raum und Zeit als unendlich und grenzenlos. Anstelle einer in ihren Zwecken vorbestimmten hierarchischen Ordnung, begegnet die Natur fortan als Ansammlung gleichwertiger einzelner Fakten, die nach selbstgesetzten Zielen durch den Menschen formbar ist. Alltagserfahrung wird nicht länger als ein Widerfahren, als Vollzug des in seinem Verlauf stets schon Bestimmten begriffen. Im Kontrast zur unhinterfragten Geltung tritt Wissen als Material ins Bewusstsein, aus dem Ziele und Methoden zur aktiven Herbeiführung neuer Erfahrungen abgeleitet werden können. Vernunft wird nicht länger einem der Erfahrung übergeordneten Reich der Ideen zugeordnet, sondern als konstruktive Intelligenz begriffen, die im Vorgang des praktischen Erfahrens der Welt operiert:
" $[\mathrm{R}]$ eason is experimental intelligence [...] It liberates man from the bondage of the past, due to ignorance and accident hardened into custom. It projects a better future and assists man in its realization. And its operation is always subject to test in expe- rience. The plans which are formed, the principles which man projects as guides of reconstructive action, are not dogmas. They are hypothesis to be worked out in practice, and to be rejected, corrected and expanded as they fail or succeed in giving our present experience the guidance it requires" (Dewey 1920, S. 96).

Ein weiterer Einstellungswandel betrifft die Vorstellung des Status von Idealen, der eng mit einer veränderten Haltung gegenüber dem Phänomen des Wandels zusammenhängt. Für Dewey ist im vormodernen Weltbild die vorübergehende und flüchtige Realität der Dinge in der phänomenalen Welt der Erfahrung der ewig unveränderlichen Ordnung im Reich des Noumenalen entgegengesetzt. Ideen und Ideale werden als unveränderliche, als jenseits der Erfahrung wahrgenommen. "This is Ideal, the Form of Forms, because it has no lacks, no needs, and experiences no change or variety" (Dewey 1920, S. 111). Philosophie, als die höchste Form der Kontemplation, die sich der Schau der Ideen annimmt, muss aus dieser Perspektive als eine in sich geschlossene, selbstgenügsame Tätigkeit erscheinen. Sie hat keinen praktischen Zweck, sondern ist im Streben nach reiner theoretischer Erkenntnis Zweck an sich.

Die Moderne dagegen versteht Wandel nicht länger als Zeichen der Unvollkommenheit, sondern als Fortschritt. Moderne Wissenschaft zielt nicht darauf ab, eine hinter beobachteten Veränderungen liegende unveränderliche Ordnung zu finden. "Rather, the experimental method tries to break down apparent fixities 
and to induce changes" (Dewey 1920, S. 113). Damit verändert sich auch die Wahrnehmung von Ideen und Idealen. Sie werden nicht länger als abseits und getrennt von praktischer Erfahrung angenommen, sondern übernehmen eine wesentliche Funktion im Zusammenhang des praktischen Welterschließens:
"When the belief that knowledge is active and operative takes hold of men, the ideal realm is no longer something aloof and separate; it is rather that collection of imagi- ned possibilities that stimulates men to new efforts and realizations. [...] [T]he pic- ture of the better is shaped so that it may become an instrumentality of action, while in the classic view the Idea belongs ready-made in a noumenal world" (Dewey 1920, S. 118).

Deweys Hinweis auf das Bewusstwerden von Welterschließung als fortdauernde Rekonstruktion menschlicher Erfahrung darf nun nicht darüber hinweg täuschen, dass für ihn der größte Teil praktischer Erfahrung unmittelbar und unreflektiert abläuft. Im Unterschied zu diesem primären Weltzugang bezieht sich die Rede von der Rekonstruktion menschlicher Erfahrung auf einen reflexiven Umgang mit der Welt, der immer erst dann auftritt, wenn routinemäßiges Handeln auf ein irgendwie geartetes Problem stößt und der Handelnde an zuvor Unhinterfragtem aktiv zu Zweifeln beginnt (vgl. Jörke 2003, S.46-54). Wie Joas beschreibt, führt Dewey zufolge aus dieser Situation des Zweifels heraus

„nur eine Rekonstruktion des unterbrochenen Zusammenhangs. Die Wahrnehmung muss neue oder andere Aspekte der Wirklichkeit erfassen; die Handlung muss an anderen Punkten der Welt ansetzen oder sich selbst umstrukturieren. Diese Rekonstruktion ist eine kreative Leistung des Handelnden. Gelingt es, durch die veränderte Wahrnehmung die Handlung umzuorientieren und damit wieder fortzufahren, dann ist etwas Neues in die Welt gekommen: eine neue Handlungsweise, die sich stabilisieren und selbst wieder zur unreflektierten Routine werden kann“ (Joas 1992a, S. 190).

In unserem Zusammenhang ist entscheidend, dass Deweys Überlegungen auf ein Ambivalent-Werden der sozialen Geltung von Idealen in gesellschaftlicher Praxis verweisen, das auch für Habermas zentral ist. Einerseits sind Ideale Vorstellungen, deren soziale Geltung eine den jeweiligen Handlungskontext überschreitende, die Akteure orientierende Wirkung hat. Andererseits wird die Gültigkeit derselben Ideale nicht - wie in der klassischen Konzeption - als absolut wahrgenommen, sondern als selbst aus vergangenen menschlichen Erfahrungen erwachsen. Wie Jörke betont, geht Dewey davon aus, dass „,unsere Welt eine Welt der Kontingenz ist, eine sich immer verschiebende Mischung aus Stabilem und Prekärem “ (Jörke 2003, S. 47). Bei Habermas bringt sich dieser Gedanke in der Annahme zum Ausdruck, dass das moderne Bewusstsein von einem fallibilistischen Sinn gezeichnet ist, der jede Tatsache und Wertvorstellung von einer Ambivalenz zwischen „Faktizität und Geltung " durchzogen erscheinen lässt. In diesem Sinne soll nun veranschaulicht werden, inwiefern sich Habermas` Theorie als eine verdichtende Weiterführung dieses Deweyschen Gedankengangs begreifen lässt. 


\section{Der Stellenwert von Rekonstruktion im Kontext politischer Theorie als Gesellschaftskritik}

Gegenwärtig spielt der Begriff der Rekonstruktion vornehmlich im Diskurs politischer Theorie als Gesellschaftskritik eine Rolle. In der Debatte um die angemessene Form einer Theorie der Gerechtigkeit, die zur Kritik politischer Verhältnisse in liberal-demokratischen Gesellschaften herangezogen werden sollte, stehen sich zwei Modelle gegenüber: konstruktive und rekonstruktive Gerechtigkeitstheorie. ${ }^{4}$ Der wesentliche Unterschied zwischen ihnen betrifft die Frage, auf welche Art ein normatives Ideal gerechter Ordnung theoretisch zu bestimmen ist, in dessen Lichte die Realität liberal-demokratischer Gesellschaften kritisch beurteilt werden kann. Axel Honneth (2011) hat jüngst eine kritische Theorie der Gerechtigkeit vorgelegt, in der er eine auf Hegel zurückgehende Methode normativer Rekonstruktion gegenüber konstruktivistischen Gerechtigkeitstheorien als überlegen auszeichnet. Hier wendet er sich unter anderem gegen die Diskurstheorie von Habermas, die er als eine Variante konstruktivistischer Gerechtigkeitstheorie betrachtet. $\mathrm{Da}$ es sich hierbei meines Erachtens um eine in der Literatur gängige verkürzte Rezeption der Habermasschen Diskurstheorie handelt, möchte ich im Folgenden zunächst die Grundzüge von Honneths Entwurf rekapitulieren (3.1). Vor diesem Hintergrund lassen sich dann die Unterschiede genauer benennen, die zwischen dem Stellenwert von Rekonstruktion in Honneths und Habermas' Theorie bestehen. Der wesentliche Punkt ist: bei Habermas steht die Methode rationaler Rekonstruktion nicht im Zusammenhang einer kritischen Gerechtigkeitstheorie, wie sie Honneth verfolgt. Nur wenn man den soziologisch-explanativen Sinn ernst nimmt, den Habermas mit den Begriffen des Diskurses sowie der Methode rationaler Rekonstruktion verbindet, lässt sich die Reichweite der Konsequenzen seiner Argumentation nachvollziehen (3.2).

\subsection{Der Stellenwert normativer Rekonstruktion in Axel Honneths Gerechtigkeitstheorie}

Honneths Argument ist motiviert durch eine Kritik an dem, was er den Siegeszug kantischer, konstruktivistisch verfahrender Ansätze in der Gerechtigkeitstheorie bezeichnet. Sein Haupteinwand gegenüber konstruktivistischen Gerechtigkeitstheorien lautet, dass sie als ideale Theorien (Rawls 1975) Prinzipien gerechter Ordnung im abstrakten Gedankenexperiment freistehend, das heißt unter Ausblendung faktischer gesellschaftlicher Verhältnisse entwerfen. Die Schwäche konstruktivistischer Gerechtigkeitstheorien resultiert daraus, nicht wissen zu können, ob sich „die Kluft zwischen normativen Forderungen und gesellschaftlicher

4 Mitunter wird ein weiteres Modell genealogischer Kritik (Saar 2009) unterschieden. Man kann jedoch mit Honneth davon ausgehen, dass es sich dabei um ein, „parasitäres Kritikverfahren“ (Honneth 2007, S. 63) handelt, welches seine vorausgesetzten normativen Maßstäbe nicht selbst begründet und daher keinen distinkten Ansatz einer Gerechtigkeitstheorie enthält, der in diesem Zusammenhang von Relevanz wäre. Eine differenzierte Diskussion verschiedener Modelle von Gesellschaftskritik findet sich bei Iser (2008). 
Realität überhaupt überbrücken lässt“ (Honneth 2011, S. 119). ${ }^{5}$ Diese Schwäche kann Honneth zufolge auch nicht dadurch behoben werden, dass Gerechtigkeitsgrundsätze in nicht-idealer Theorie nachträglich mit der gesellschaftlichen Realität konfrontiert werden, um realisierbare Wege zu gerechteren Gesellschaftszuständen zu ermitteln. ${ }^{6}$ Das Problem idealer wie nicht-idealer Theorie liegt für Honneth in der vorgestellten Arbeitsteilung zwischen Gerechtigkeitstheorie einerseits und der in der Sozialwissenschaft vollzogenen Gesellschaftsanalyse andererseits, die einen Bruch impliziert: Unter Absehung von gesellschaftlichen Tatsachen konstruierte Gerechtigkeitsprinzipien lassen sich anhand einer sozialwissenschaftlichen Beschreibung gesellschaftlicher Realität, die von für Gerechtigkeitsfragen relevanten normativen Zusammenhängen keine Notiz genommen hat, weder als realistisch noch als unrealistisch ausweisen. Auch in nicht-idealer Theorie wird „eine von dritter Seite aufbereitete und bestimmte Realität vorausgesetzt [...], auf die dann erst im nachhinein die normativen Maßstäbe appliziert werden“ (Honneth 2011, S. 22). Auf die Überwindung dieses Bruchs „zwischen Begründung und Anwendung " (Honneth 2011, S. 105) zielt Honneth mit seinem Vorschlag, die Grundsätze gerechter Ordnung anhand der Ansprüche, die sich in liberaldemokratischen Gesellschaften historisch ausgebildet haben, normativ zu rekonstruieren.

Normative Rekonstruktion bezeichnet bei Honneth also eine Methode der Herleitung einer angemessenen Konzeption gerechter Gesellschaftsordnung, die in der Lage sein soll, praktische Motivationskraft zu entfalten. Angemessen ist eine Konzeption der Gerechtigkeit demnach dann, wenn sie aus dem systematischen Nachvollzug des normativen Sinns etablierter Praktiken rekonstruiert ist und daher als historisch wirksam gelten kann. Die Betonung liegt hier auf systematischem Nachvollzug. Für Honneth beschränkt sich rekonstruktive Gerechtigkeitstheorie nicht auf eine bloße „Interpretation“ von Handlungspraktiken (Honneth 2007, S. 64-67), die einer „hermeneutischen Rückanpassung“ (Honneth 2010, S. 16) ihrer Prinzipien an existierende Institutionengefüge und damit einer Affirmation des Bestehenden gleichkommt. ${ }^{7}$ Ihre Besonderheit besteht in Folgendem: „Die gegebenen Institutionen und Praktiken werden auf ihre normativen Leistungen hin in der Reihenfolge analysiert und dargestellt, in der sie für die soziale Verkörperung und Verwirklichung der gesellschaftlich legitimierten Werte von Bedeutung sind." (Honneth 2011, S. 23). ${ }^{8}$

Damit ist nun klar, dass Honneths Gerechtigkeitstheorie genau genommen aus zwei Stufen besteht. Sie leitet zunächst einen gesellschaftlich legitimierten Grundwert als „normativen Bezugspunkt“ (S. 120) her, um dann über eine normativrekonstruktive Gesellschaftsanalyse aufzuzeigen, welche Bedeutung bestimmten etablierten Institutionen zur Verwirklichung dieses Werts zukommt und in welchem Maße die in ihnen institutionalisierten „Praxispotentiale“ (S. 27) tatsäch-

5 Einen ähnlichen Einwand hat Raymond Geuss (2008) jüngst gegen politische Theorien in der kantischen Tradition vorgebracht.

6 Zum Verhältnis von idealer und nicht-idealer Theorie vgl. Schaub (2010) und Simmons (2010).

7 In dieser Hinsicht wendet sich Honneth von sogenannten immanenten Gerechtigkeitstheorien ab, wie sie etwa Walzer (1992) oder MacIntyre (1987) vertreten.

8 Nachfolgende Seitenangaben beziehen sich auf diesen Titel. 
lich wirksam geworden sind. Auf der ersten Stufe, der Bestimmung des Grundwerts moderner Gesellschaften, geht Honneth nun zunächst von verschiedenen Prämissen aus, die nicht weiter erläutert werden. Erstens setzt er voraus, dass in modernen Gesellschaften „ein einziger Wert die Legitimationsgrundlage der sozialen Ordnung bildet" (S. 122), nämlich der Wert gleicher individueller Freiheit. Die zweite Prämisse ist, dass der Begriff individueller Freiheit mit dem der Gerechtigkeit in einem internen Zusammenhang steht (S. 39). Drittens hat sich diese Legierung von Autonomie und Gerechtigkeit als Resultat eines historischen Lernprozesses zum Kern des moralisch-praktischen Bewusstseins der Moderne entwickelt (S. 40). Diese Definition des Grundwerts moderner Gesellschaften ist Honneth zufolge aber noch zu unbestimmt, um aus ihr Bedingungen einer gerechten Sozialordnung abzuleiten. Stattdessen muss die Idee gleicher individueller Freiheit nach den drei typischen Deutungen, die sie im modernen politischen Denken erfahren hat, differenziert werden: dem Begriff negativer, reflexiver und Hegels Begriff sozialer Freiheit. Honneth geht es dabei nicht darum, einen der Freiheitsbegriffe zugunsten eines anderen aufzugeben. Vielmehr zeigt er auf, dass die Begriffe negativer und reflexiver Freiheit, die den meisten konstruktivistischen Gerechtigkeitstheorien zugrunde liegen, keine selbstständigen Freiheitsbegriffe sind. Sie bilden vielmehr - zusammen mit sozialer Freiheit - jeweils einen unerlässlichen Aspekt dessen, was insgesamt erst den Wert individueller Freiheit ausmacht. Dennoch hält Honneth soziale Freiheit in einem anderen Sinn für grundlegend. Sie liegt nämlich immer dann vor, wenn Individuen in ungezwungenen Verhältnissen der wechselseitigen Anerkennung gemeinsam handeln. Während soziale Freiheit also gewissermaßen den Normalzustand der ungezwungenen Hervorbringung von Gemeinschaft benennt, stellen negative und reflexive Freiheit lediglich Ausfallbürgschaften dar. Indem sie dem Individuum ermöglichen, sich dem Zwang gestörter Anerkennungsverhältnisse zu entziehen (negative Freiheit) bzw. diesen über das Einfordern von Rechtfertigungen entgegenzutreten (reflexive Freiheit), erfüllen sie eine Schutz- bzw. Korrekturfunktion für Zusammenhänge der Vergemeinschaftung, die andernorts hervorgebracht werden.

Auf der zweiten Stufe seiner Gerechtigkeitstheorie vollzieht Honneth nun eine Gesellschaftsanalyse unter der Annahme, dass jeweils einer dieser Aspekte des Grundwerts individueller Freiheit in unterschiedlichen gesellschaftlichen Praxiszusammenhängen historisch wirksam geworden ist. Die normative Rekonstruktion zielt darauf, „die Bedingungen der Gerechtigkeit im Sinne einer schrittweisen Herauspräparierung derjenigen Handlungssphären in den liberaldemokratischen Gesellschaften der Gegenwart zu entfalten, in denen der Wert der individuellen Freiheit auf je spezifische, funktionstypische Weise institutionelle Gestalt angenommen hat" (S. 124).

Der Begriff negativer Freiheit hat sich demnach als rechtliche Freiheit im $\mathrm{Zu}$ sammenhang der positiven Rechtsordnung etabliert, der Begriff reflexiver Freiheit verkörpert sich im gesellschaftlichen Moralverständnis. Im Gegensatz zu konstruktivistischen Gerechtigkeitstheorien sind jedoch weder mit rechtlicher, noch mit moralischer Freiheit die gesellschaftlichen Existenzbedingungen individueller Frei- 
heit in liberalen Demokratien erfüllt. ${ }^{9}$ In beiden Kontexten ist vorausgesetzt, dass Individuen sich als Teilnehmer sozialer Praktiken begreifen, aus denen sie sich qua rechtlicher bzw. moralischer Freiheit gegebenenfalls befreien können. In familiären, freundschaftlichen sowie Liebesbeziehungen, im kapitalistischen Handlungssystem und in der demokratischen Willensbildung politischer Öffentlichkeit haben sich Kooperationssphären sozialer Freiheit institutionalisiert, die weder über rechtliche noch moralische Praktiken generiert werden können. Honneths kritischer Schluss lautet, dass charakteristische zeitgenössische Pathologien auf den Umstand zurückzuführen sind, dass Gesellschaftsmitglieder rechtliche und moralische Freiheit nicht als Ausfallbürgschaften sozialer Freiheit, sondern als Inbegriff individueller Freiheit verstehen. Weil aus diesem „tiefsitzenden Hang zum gesellschaftlichen Selbstmissverständnis" (Honneth 2011, S. 223) Einstellungen und Verhaltensweisen hervorgehen, die dem eigentlichen Sinn von Recht und Moral - der Ermöglichung ungezwungener gemeinschaftlicher Praxis - zuwiderlaufen, bedarf es der aufklärerischen Diagnose rekonstruktiver Gerechtigkeitstheorie.

\subsection{Der Stellenwert der Methode rationaler Rekonstruktion in der Habermasschen Theorie}

Vor diesem Hintergrund lässt sich nun der Stellenwert rationaler Rekonstruktion in Habermas' Theoriegebäude kontrastiv bestimmen. Honneth kritisiert die Habermassche Diskurstheorie dafür, den Blick auf die Kategorie des positiven Rechts zu verengen und damit die Praxissphären gesellschaftlicher Sittlichkeit als soziale Bedingungen der Gerechtigkeit außer Acht zu lassen. Diese Kritik irritiert insofern, als viele von Honneths zentralen Thesen - über den normativen Sinn demokratischer Willensbildung, dessen Abhängigkeit von ungestörten Kommunikations- bzw. Anerkennungsverhältnissen ${ }^{10}$ sowie die Gefahr gesellschaftlichen Sinnverlustes durch einseitige Wahrnehmung der doppelten Funktion demokratisch gesetzten Rechts - in weiten Teilen dem Inhalt nach mit Habermas' Erläuterungen übereinstimmen. ${ }^{11}$ Andererseits entspricht Honneths Einschätzung einer gängigen Rezeption, die die Habermassche Diskurstheorie als Theorie gerechter politischer Ordnung in gesellschaftskritischer Absicht deutet. Das ist insofern unglücklich, als damit leicht der soziologisch-explanative Geltungssinn, der sich mit der Diskurstheorie auch verbindet, ausgeblendet wird.

Tatsächlich beansprucht Habermas' Diskurstheorie des demokratischen Rechtsstaats wie Honneths Gerechtigkeitstheorie unter anderem, das moralischpraktische Selbstverständnis moderner Gesellschaften nachzuvollziehen. Der entscheidende Unterschied liegt jedoch in der Funktion, die dieses Unterfangen im

9 In Habermas (1992) liegt für Honneth ein Beispiel für eine Reduzierung der Bedingungen der Gerechtigkeit auf rechtliche Freiheit, in Forst (2007) ein Beispiel für eine auf moralische Freiheit verkürzte Gerechtigkeitstheorie vor.

10 Honneth verwendet das weitgehend synonym. Auf den Zusammenhang zwischen den Begriffen Kommunikations- und Anerkennungsverhältnis hat Iser (2008) hingewiesen.

11 Anders ergäbe beispielsweise Habermas“ zentrale These von der Tendenz einer „Kolonialisierung der Lebenswelt“ (1981b) in gegenwärtigen liberalen Demokratien, die in „Faktizität und Geltung “ (1992) nicht aufgehoben, sondern spezifiziert wird, gar keinen Sinn. 
jeweiligen Theoriekontext spielt. Während Honneth damit eine praktische Kritik an pathologischen Zuständen in liberalen Demokratien vorbereitet, ist es für Habermas ein wesentlicher Baustein im Rahmen einer Erklärung sozialer Ordnung in modernen westlichen Gesellschaften. Dieser Unterschied im Argumentationsziel ist in seiner Bedeutung kaum zu überschätzen. Obwohl er keinen Widerspruch zu Honneths Argumentation erzeugt, kehrt er die Richtung der These um: nicht weil der demokratische Rechtsstaat die "Teilnahme an diskursiven Veranstaltungen“ (Honneth 2011, S. 80) ermöglicht, stellt er eine Bedingung der Gerechtigkeit dar. Die These der Diskurstheorie lautet: weil soziale Praktiken sprachlich vermittelte Prozesse intersubjektiver Welterschließung sind, die als solche stets im Schatten der Diskursstruktur der Rechtfertigungspraxis ablaufen, hat sich über die Geschichte sozialer Kämpfe und kognitiver Lernprozesse ein moralisches und epistemisches Bewusstsein ausgebildet, das als Erklärung dafür dienen kann, warum sich die Institutionen des demokratischen Rechtsstaats in westlichen Gesellschaften erfolgreich etabliert haben und bis heute für den Erhalt sozialer Ordnung maßgeblich sind. Diese These wirkt wie ein Brennglas, in dem die verschiedenen Theoriebausteine des Habermasschen Werks zusammenlaufen (Formalpragmatik, Theorie sozialer Evolution, Moraltheorie, Erkenntnis- bzw. Wahrheitstheorie sowie Rechts- und Demokratietheorie).

Unbestritten sind Habermas und Honneth von einer ähnlichen Motivation geleitet. Beide erhoffen sich, mit ihren Theorien letztlich eine praktische Wirkung zu erzeugen. Auch Habermas geht es darum, „das normative Selbstverständnis der Moderne gegen die Gebildeten unter seinen Verächtern [zu] verteidigen “ (Habermas 2000, S. 13). Der entscheidende Unterschied ist, dass Habermas zu diesem Zweck die Entwicklung einer Theorie mit soziologisch-explanativer Überzeugungskraft für unerlässlich hält. Und diese Sichtweise wiederum kann als Folge einer grundlegenden Überzeugung verstanden werden, die Habermas mit Dewey teilt: reflexiv gewordene moderne Gesellschaften wissen letztlich darum, dass Welterschließung als eine kontinuierliche Problemlösungspraxis zu verstehen ist, die eine fortlaufende Rekonstruktion menschlicher Erfahrung mit sich bringt. Das bedeutet auch, sie reflektieren den ambivalenten Status von Idealen zwischen „Faktizität und Geltung“ - nämlich, einerseits als unangezweifelte Wertvorstellungen in der Praxis orientierende Funktion zu haben und doch andererseits in ihrer Geltung stets potentiell der historischen Transformation zu unterliegen. Daraus ergibt sich nun für Habermas an Honneths Theorie folgende Schwierigkeit: ${ }^{12}$ Wenn Dewey Recht hat, dann reicht es für eine kritische Gerechtigkeitstheorie zur normativen Rechtfertigung einer Ordnung nicht mehr aus, ohne nähere Begründung darauf zu verweisen, „ein höheres Maß an Übereinstimmung mit vortheoretischen Intuitionen“ (Honneth 2011, S. 113) aufzuweisen, will sie moderne Adressaten mit einem reflexiven Bewusstsein überzeugen und motivieren. Reflexiv

12 Werksgeschichtlich hat sich die nachfolgende Überlegung Habermas' natürlich nicht an der erst später vorgelegten Theorie Honneths entfaltet, sondern bereits Anfang der 1970er-Jahre. Sie stellt einen wesentlichen Aspekt dessen dar, was man als „linguistische“ oder „pragmatische Wende“ im Habermasschen Werk bezeichnen könnte (vgl. dazu Habermas 1973, S. 411-417; 1981a, S. 7-44). Die folgende Auseinandersetzung zwischen Honneth und Habermas simuliere ich lediglich, um den Punkt anschaulicher zu machen. 
gewordene Gesellschaften wissen, dass sich ihr Selbstverständnis - also geteilte vortheoretische Intuitionen - in fortlaufender Transformation befindet. Daher stellt sich ihnen die Frage: Selbst wenn die Institutionen des demokratischen Rechtsstaats unserem gegenwärtigen Moralbewusstsein entsprechen, welchen Grund haben wir zu der Annahme, dass die Ideale des modernen Moralbewusstseins und, in der Folge, eine entsprechende Institutionenordnung, nicht längst veraltet sind und zur Lösung politisch-praktischer Probleme nach wie vor angemessene Orientierung bieten? Zur Beantwortung dieses Zweifels sieht Habermas eine Theorie als notwendig an, die zweierlei erklären können muss: erstens muss sie eine Erklärung dafür anbieten, warum die Begründung der Praxis demokratischer Rechtsstaaten keinen Zufall darstellt, sondern aus der Perspektive der historisch Beteiligten als rational, also als dauerhafte Bewältigung des gesellschaftlichen Problems sozialer Ordnung unter den Bedingungen der Gerechtigkeit erscheinen musste. Zweitens muss sie aufzeigen können, dass sich das Problem sozialer Ordnung in den relevanten Aspekten heute nach wie vor so stellt wie für die damals Beteiligten.

Habermas fängt also gewissermaßen dort an, wo Honneths Theorie aufhört. Seine Theorie zielt auf eine Erklärung für das, was Honneth als Prämissen voraussetzt: zum einen, dass gleiche individuelle Freiheit sich nicht zufällig zum Grundwert des zeitgenössischen Bewusstseins entwickelt hat, sondern als Ergebnis eines Lernprozesses zu begreifen ist; und zum anderen, dass die historische Begründung der Ordnung des demokratischen Rechtsstaats vor diesem Hintergrund als eine rationale Problemlösung erklärt werden kann. Die Schwierigkeit ist dabei folgende: Wie lässt sich die Annahme eines historischen Lernfortschritts mit der pragmatistischen Einsicht vereinbaren, dass Wertvorstellungen nicht metaphysisch gegeben sind, sondern in welterschließender Praxis fortlaufend hervorgebracht werden? Die gesuchte Erklärung muss einen Standard für die Rationalität gesellschaftlichen Bewusstseins ausweisen, der es erlaubt, die historische Transformation menschlicher Erfahrung als gerichtet vorzustellen, dabei aber selbst weder als historisch kontingente noch als metaphysische Wertvorstellung erscheint. Einen solchen Rationalitätsstandard kann man Habermas zufolge den Ermöglichungsbedingungen sprachlich vermittelter welterschließender Praxis entnehmen. Die zentrale These ist: Weil Sprache alternativlos als Medium der gemeinsamen Welterschließung fungiert und weil ein Sprecher jedes Mal, wenn er sich mit jemandem über etwas verständigt, automatisch bestimmte idealisierende Unterstellungen vornimmt (und vornehmen muss), ist jede historische Interaktionssituation unvermeidlich mit Geltungsansprüchen verschiedener Art unterlegt (dazu Habermas 2005, S. 27-57). Diese Idealisierungen im Vorgang des Sprachgebrauchs, die Habermas mit dem Begriff Diskursstruktur zusammenfasst, sind keine moralischen Ideale. ${ }^{13}$ Sie wirken nicht als präskriptives Muss, dem man aus moralischen Erwägungen heraus folgen könnte oder nicht. Vielmehr handelt es sich bei ihnen

13 Obwohl beide Ansätze ansonsten starke Parallelen aufweisen, unterscheidet sich an diesem theoriearchitektonisch tiefliegenden Punkt der Habermassche Grundbegriff des Diskurses von Rainer Forsts Grundbegriff eines „Rechts auf Rechtfertigung”. Vgl. zu dieser Auseinandersetzung Forst (2011) und Habermas (2012, S. 294-298). 
um Unterstellungen, die man nicht vermeiden kann, wenn man die Praxis gemeinsamer Verständigung vollzieht. Als Bedingung jeder Art von Rationalität ${ }^{14}$ bilden sie ein und dasselbe innerweltliche Fundament, aus denen sich die Begriffe moralischer Richtigkeit, ethischer Angemessenheit sowie der Begriffs eines wahren Sachverhalts erst ableiten. Habermas erläutert somit die elementare Diskursstruktur des Sprachgebrauchs als Motor eben jener historischen Dynamik, die Dewey als fortlaufende Rekonstruktion menschlicher Erfahrung bezeichnet.

Für uns kommt es nun darauf an, den Stellenwert dieser Annahme eines „kantischen Pragmatismus“ (Habermas 1999a, S. 40) sowie die Methode der rationalen Rekonstruktion im Kontext der Habermasschen Theorie klar vor Augen zu führen. Habermas bezeichnet das Vorgehen, am Beispiel typischer sprachlicher Interaktionssituationen die stillschweigend und unvermeidlich vorgenommenen, idealisierenden Unterstellungen der Beteiligten vom Impliziten ins Explizite zu überführen, als rationale Rekonstruktion. Diese rationale Rekonstruktion von Sprecherkompetenzen ist ein Baustein im übergeordneten Argumentationszusammenhang. Ziel ist ja, aus der modernen Intuition, der Grundwert gleicher individueller Freiheit stelle eine „nur um den Preis der kognitiven Barbarei noch einmal rückgängig zu machende Errungenschaft" (Honneth 2011, S. 39) dar, eine Einsicht zu machen, die auf einer theoretischen Erklärung ruht. In diesem Zusammenhang stützt die rationale Rekonstruktion von Sprecherkompetenzen aber nicht den Schluss, die kommunikative Vernunft erzwinge einen gesellschaftlichen Lernprozess, der unaufhaltsam in Richtung eines mit ihren Idealisierungen identischen moralischen Bewusstseins führe. ${ }^{15}$ Derartige geschichtsteleologische Argumentationen haben in der reflexiv gewordenen Moderne jeden Halt verloren. Habermas' Argument lautet anders: Wenn die formalpragmatische Rekonstruktion zutrifft, kann man davon ausgehen, dass die Diskursstruktur des Sprachgebrauchs als Ermöglichungsbedingung der Welterschließung in jeder historischen Interaktionssituation im Hintergrund wirksam ist. Sie hält die Subjekte in Form einer „schwachen transzendentalen Nötigung“ (Habermas 1992, S. 18) dazu an, das in der Handlungssituation stillschweigend vorausgesetzte Tatsachenwissen sowie ethische Überzeugungen im Lichte der geteilten Situation immer dann auf ihre Geltung hin zu überprüfen, wenn ein Verständigungsproblem auftritt. Sie macht sich für jedermann als irritierender Stachel kognitiver Dissonanzen bemerkbar. Trifft das zu, kann dieser Umstand als Motor einer historischen „Dynamik des Wissenszuwachses durch Problemlösung und Rechtfertigung “ (Habermas 1999a, S. 36) aufgefasst werden, der sich gleichwohl in seinem tatsächlichen Verlauf nicht voraussehen lässt. Retrospektiv lässt sich damit aber die Hypothese begründen, dass die Geschichte moderner Gesellschaften als ein Transformationsprozess kollektiver Deutungsroutinen - des gesellschaftlichen Bewusstseins - zu vermuten ist, der sich unter den Bedingungen dieser Dynamik vollzogen hat und daher keine beliebige Transformation ist. Freilich fehlt bis zu dieser Stelle noch der entscheidende Schritt, der aus dieser Argumentation eine Erklärung der Ausgangsfrage

14 Für eine Kritik dieser Annahme vgl. Schnädelbach (1992).

15 Wie Habermas' Formulierung eines mit dem Sprachgebrauch aufgerufenen „Telos“ der Kommunikation auf den ersten Blick vermuten lassen könnte. 
macht. Um zu begründen, dass das moderne normative Selbstverständnis als Ergebnis eines gesellschaftlichen Lernprozesses begriffen werden kann, muss nun gezeigt werden, dass die Reihenfolge der wesentlichen Transformationen historischer Bewusstseinslagen tatsächlich einen epistemischen Richtungssinn aufweist, der zu dem Richtungssinn der im Hintergrund sprachlicher Verständigungspraxis vorgenommenen Idealisierungen parallel verläuft. Erst dann liegt eine theoretische Rechtfertigung dafür vor, die historischen Bewusstseinstransformationen als einen Rationalisierungsprozess zu begreifen, der im modernen, reflexiv gewordenen Bewusstsein seinen nur vorläufigen Endpunkt gefunden hat.

Damit lässt sich nun der Stellenwert rationaler Rekonstruktion bei Habermas im Unterschied zur kritisch-aufklärerischen Funktion der normativen Rekonstruktion in Honneths Gerechtigkeitstheorie festhalten. An dieser Stelle gilt es, zwei weit verbreiteten Deutungen entgegenzutreten. Zum einen besteht der Zweck der rationalen Rekonstruktion von Sprecherkompetenzen nicht in der Etablierung eines universalen Maßstabs des moralisch Richtigen, der „in der Sprache angelegt“ ist (Schweppenhäuser 2005, S. 229; Fuchs-Goldschmidt 2008) 16 $^{16}$ und eine praktische Kritik gesellschaftlicher Verhältnisse vorbereiten soll. ${ }^{17}$ Die Explikation der normativen Orientierungen, die als Bedingungen problemlösenden Handelns (stillschweigend) im Hintergrund empirischer Interaktionssituationen wirksam sind, erfüllt stattdessen einen zweifachen epistemischen Zweck. Für sich genommen beansprucht sie, das, was wir als Sprecher in einer Interaktionssituation (immer auch) tun, nachzuvollziehen. Darüber hinaus erfüllt die formalpragmatische Rekonstruktion aber eine wichtige Funktion in einem übergeordneten Erklärungszusammenhang. Sie dient zur Entwicklung einer Hypothese über die Dynamik des Verlaufs historischer Praxis, die nun dadurch bestätigt werden muss, dass man sie als „Input[...] in empirischen Theorien verwendet“ (Habermas 1983b, S. 41). Habermas‘ Theorie sozialer Evolution ${ }^{18}$ sowie die Diskurstheorie des demokratischen Rechtsstaats schließen genau hier an. Sie analysieren die Abfolge „der institutionellen Verkörperungen innovativer Bewusstseinsstrukturen in der Geschichte“ (Habermas 1983b, S. 41) und sollen aufzeigen, dass diese Abfolge sich im Sinne der Hypothese als Rationalisierung beschreiben lässt. Das steht einer zweiten Lesart entgegen, nach der der Gegenstandsbereich rationaler Rekonstruktion bei Habermas auf universale Kompetenzen sprechender und handelnder

16 Sie hat meines Erachtens auch nicht den Zweck zu zeigen, dass unsere moralischen Intuitionen in etwas Universalem wurzeln („rooted“) (McCarthy 1994, S. 47). Habermas nimmt vielmehr den über die Kommunikationsform rationaler Diskurse freigesetzten Begründungsbedarf als Katalysator moralischer Lernprozesse an (Habermas 1999b, S. 296-307). „So erklärt sich die Universalität einer Welt wohlgeordneter interpersonaler Beziehungen - der Entwurf eines moralischen Universums auf das hin argumentiert wird - aus der Widerspiegelung des egalitären Universalismus, auf den sich Argumentationsteilnehmer immer schon einlassen müssen, wenn ihr Unternehmen nicht seinen kognitiven Sinn einbüßen soll.“ (Habermas 1999b, S. 307)

17 In diesem Sinne wird die Rekonstruktion der Verständigungspraxis mitunter als theoretische Folie verstanden, mit der Habermas eine „Beurteilung praktischer Fragen“ (Flügel 2008, S. 117) vorbereitet bzw. für eine „Stärkung der intersubjektiven Verständigung in all ihren Dimensionen“ (Iser 2008, S. 89) argumentiert. Diese Interpretationen mögen ein grundsätzliches Anliegen von Habermas treffen, sie erfassen jedoch meines Erachtens nicht den Geltungsanspruch seiner Theorie.

18 Habermas' Theorie sozialer Evolution kann ich hier nicht diskutieren. Vgl. dazu Gaus (2009, S. 148-233). 
Subjekte eingeschränkt sei. ${ }^{19}$ Wie ich nun illustrieren möchte, stellt die Diskurstheorie des demokratischen Rechtsstaats selbst eine rationale Rekonstruktion dar, die im Zusammenhang des Habermasschen Theoriegebäudes mit einem soziologisch-explanativen Geltungsanspruch auftritt.

\section{Zur Bedeutung rationaler Rekonstruktion für die empirisch verfahrende Analyse politischer Praxis}

Um die Bedeutung des Habermasschen Begriffs der Rekonstruktion im Kontext empirisch verfahrender Analyse besser zu verstehen, ist es hilfreich, die Diskurstheorie des demokratischen Rechtsstaats als einen zweiten Anwendungskontext der Methode rationaler Rekonstruktion in Habermas' Theorie zu verstehen. Dort geht es nicht um den Nachvollzug allgemeiner Bedingungen der Möglichkeit von Rationalität, sondern um die Rekonstruktion bestimmter historisch ausdifferenzierter Rationalitätsstrukturen, die sich in der modernen Institutionenordnung des demokratischen Rechtsstaats verkörpert finden. Um das nachzuvollziehen, gilt es zunächst, sich der grundlegenden soziologischen Bedeutung zu vergewissern, die Habermas dem Begriff rationaler Rekonstruktion beimisst. Obwohl von Habermas nicht explizit herausgearbeitet, verbinden sich mit dem Begriff Überlegungen, die auf ein allgemeines Modell sozialwissenschaftlicher Erklärung zielen. ${ }^{20}$ Das soll zunächst in wenigen Umrissen skizziert werden (4.1). Vor diesem Hintergrund wird dann deutlich, inwiefern sich Habermas' Diskurstheorie des demokratischen Rechtsstaats nicht primär als Gesellschaftskritik, sondern als ein Beitrag zu einer „rekonstruktiv verfahrende[n] Soziologie der Demokratie“ (Habermas 1992, S. 349) verstehen lässt (4.2). ${ }^{21}$ Akzeptiert man die diskurstheoretischen Annahmen, so ergeben sich daraus Folgen für die Analyse politischer Praxis, die schließlich am Beispiel der empirischen Legitimationsforschung illustriert werden sollen (4.3).

19 Vgl. dazu die differenzierten Überlegungen zu Habermas' Methode rationaler Rekonstruktion von Celikates (2009) und Pedersen (2008). Meine Differenzen zu Celikates und Pedersen beschränken sich im Wesentlichen auf diesen Punkt, der jedoch meines Erachtens für das Verständnis der methodischen Implikationen der Diskurstheorie für die empirisch orientierte Politikwissenschaft entscheidend ist.

20 Garz (2000) hat zu Recht darauf hingewiesen, dass im Hinblick auf die explizite Ausarbeitung einer Methode in Habermas، Theoriegebäude eine Forschungslücke besteht. Daraus folgt aber meines Erachtens nicht, dass Habermas' Ansatz „in einer durch das Auftauchen qualitativ-empirischer Methoden sich verändernden Forschungslandschaft an Einfluss verlieren“ (Garz 2000, S. 201) muss. Nicht nur deshalb, weil - wie Garz selbst am Beispiel von Oevermanns (2000) Methodologie der objektiven Hermeneutik anschaulich darlegt - sich eine Verbindung zu weiter ausgearbeiteten soziologischen Analysemethoden herstellen lässt. Sondern auch, weil - entgegen Garz' These - die Beschäftigung mit Gegenständen der sozialwissenschaftlichen Forschung bei Habermas keine „Leerstelle“ (Garz 2000, S. 208) bildet. Wie nachfolgend gezeigt werden soll, thematisiert Habermas grundlegende Aspekte der Analyse politischer Praxis nicht nur methodologisch, sondern begreift seine Theorie des demokratischen Rechtsstaats selbst als einen Beitrag in dieser Hinsicht.

21 Das bedeutet nicht, wie oben ausgeführt, dass die Diskurstheorie des demokratischen Rechtsstaats im Kontext der Gesellschaftskritik keine Rolle spielen könnte. Im Gegenteil. Mir kommt es hier aber darauf an, ihren soziologisch-explanativen Geltungsanspruch zu betonen, der in der Rezeption meist unbeachtet bleibt. Vgl. z. B. Forst (1999); Gutmann u. Thompson (2001); Rehg u. Bohman (2002); Schmalz-Bruns (1995). 


\subsection{Rationale Rekonstruktion als Modell sozialwissenschaftlicher Erklärung}

Habermas nimmt an, dass nicht die isolierte Handlung eines Individuums, sondern der Ablauf einer sozialen Interaktion als kleinste Untersuchungseinheit betrachtet werden muss (Habermas 1988, S. 68-69). Eine einzelne Handlung ist stets Reaktion auf vorgängige und einschränkende bzw. ermöglichende Bedingung nachfolgender Handlungen. Entsprechend versteht man die Bedeutung eines Ereignisses, wenn man versteht, in welchem Sinn eine Handlung an einen vorherigen Handlungskontext anschließt. Habermas' Handlungstheorie ist eine Theorie der Handlungskoordination. Damit verbindet sich eine methodologische Perspektivenverschiebung vom Motiv des Akteurs zur Rationalität von Handlungsanschlüssen. An die Stelle, die die individuelle Präferenzordnung im Zusammenhang einer intentionalen Erklärung einnimmt, tritt die jeweils von den Beteiligten ad hoc, meist unreflektiert vollzogene Deutung der gemeinsam erlebten Situation. Um auf die Gründe schließen zu können, aus denen die beteiligten Akteure gehandelt haben, muss die Analyse der „symbolic sphere that serves as the background of these specific reasons" (Peters 1996, S. 120) mit einbezogen werden. Diese Refokussierung überwindet insbesondere zwei Annahmen, die für viele politikwissenschaftliche Analyseansätze zentral sind.

Erstens wird dadurch das Spektrum der möglichen Rationalität einer politischen Interaktion über die eigeninteressierte strategische Verfolgung subjektiver Interessen hinaus erweitert. Gegen einen solchen eindimensionalen Begriff von Handlungsrationalität, der mitunter in ökonomischen Rational Choice Theorien unterstellt wird (vgl. Schmalz-Bruns u. Hitzel-Cassagnes 2003), hat sich auch der akteurzentrierte Institutionalismus (Mayntz u. Scharpf 1995) gewendet. Dort wird ein spieltheoretisches Erklärungsmodell vorgeschlagen, das dem Kontext politischer Praxis insofern angemessener ist, als es die Kategorie individueller Präferenzen nicht auf Eigeninteresse beschränkt, sondern um normative Rollenerwartungen sowie Akteuridentität erweitert (Scharpf 2000, S. 116-121). Während die Erklärungskraft des akteurzentrierten Institutionalismus im Bereich von PolicyAnalyse außer Zweifel steht, ergibt sich aus diskurstheoretischer Sicht eine wichtige Einschränkung. Der akteurzentrierte Institutionalismus schließt aus der Erweiterung des Rationalitätsspektrums im politischen Handeln, dass individuelle Präferenzen nicht als determiniert unterstellt werden können. Vielmehr ist der Inhalt der Präferenzordnungen der Beteiligten jeweils fallspezifisch empirisch zu bestimmen, bevor das Ergebnis eines Policy-Prozesses als Resultat strategisch interdependenten Handelns der Beteiligten erklärt werden kann. Diese Offenheit wird jedoch andererseits durch die Unterstellung eingeschränkt, dass ein ganz bestimmter Aspekt die Präferenzordnung der Akteure im Normalfall dominiert. Sowohl das Eigeninteresse als auch die normativen Beschränkungen im politischen Handeln werden aus den Regeln des jeweiligen institutionellen bzw. organisatorischen Kontextes abgeleitet, in dem die Akteure handeln (Scharpf 2000, S. 81-82).

Damit hält der akteurzentrierte Institutionalismus an einer zweiten Prämisse fest, die die Diskurstheorie vermeidet: der Unterstellung, dass sich die Rationalität empirischer politischer Praxis theoretisch vorbestimmen lässt. Zwei Aspekte sprechen gegen diese Unterstellung. Zum einen legen empirische Studien zur Theorie 
deliberativer Demokratie nahe, dass individuelle Präferenzen im Verlaufe politischer Interaktionen nicht stabil bleiben, sondern von (teils institutionell bedingten) Prozessen kommunikativer Verständigung begleitet sind, die in unterschiedlicher Weise den Wandel politischer Präferenzen erwirken (Landwehr 2009). Zum anderen handelt es sich bei politischen Institutionen um „living institutions“, die selbst in einem eigentümlichen Spannungsverhältnis zwischen „change and continuity " (Olsen 2010) stehen. Sie wirken daher einerseits normativ strukturierend auf Interaktionszusammenhänge ein, unterliegen aber zugleich selbst fortlaufend Transformationsprozessen. Auch politische Ordnungszusammenhänge sind von der Dynamik erfasst, die Dewey als kontinuierliche Rekonstruktion menschlicher Erfahrung bezeichnet. In diesem Licht besehen, nimmt der akteurzentrierte Institutionalismus politische Praxis unter ganz bestimmten Kontextbedingungen in den Blick - im Kontext einer stabilen politischen Ordnung. Dagegen bedarf es zur Erklärung politischer Interaktion in ungefestigten, sich transformierenden politischen Ordnungen (beispielsweise im Kontext der EU) einer offeneren Analyse, die sich kontextsensibel an den jeweiligen empirischen Interaktionskontext anschmiegen kann. Hier ist beispielsweise von der Möglichkeit auszugehen, dass die politischen Akteure den institutionellen Kontext selbst als in Transformation befindlich wahrnehmen und ihr Handeln zugleich von der gegenwärtigen politischen Ordnung orientiert und auf die aktive Transformation derselben gerichtet ist.

Habermas' Begriff rationaler Rekonstruktion als Modell sozialwissenschaftlicher Erklärung ist hier offener, weil er die Situationsgebundenheit politischer Interaktion in den Vordergrund stellt. Zum Verständnis der Rationalität einer Interaktion bedarf es deshalb rekonstruktiver Verfahren, weil sich die Gründe für die beobachteten Handlungen nur im Lichte der Situationsdeutungen ermitteln lassen, die die jeweils Beteiligten in ihrem empirischen Handlungskontext mehr oder weniger stillschweigend vorgenommenen haben. Bei der theoretischen Nachkonstruktion von Situationsdeutungen in politischer Praxis darf nun zweierlei nicht außer Acht gelassen werden. Erstens weist Habermas daraufhin, dass in politischer Praxis neben Eigeninteresse und institutionalisierten Ordnungen eine dritte Rationalitätsebene wirksam ist - das, was man als den Hintergrund gesellschaftlich geteilter kognitiver und moralischer Überzeugungen oder auch als gesellschaftliches Bewusstsein bezeichnen könnte (vgl. Peters 1996, S. 107). Diese Ebene ist insbesondere im Kontext ungefestigter oder zur Erklärung sich herausbildender politischer Ordnung von Bedeutung. Politische Ordnungen sind insofern in tieferliegende „narrative frames of plausibility“ (Eder 2009, S. 70) eingebettet, als ihre dauerhafte Stabilität davon abhängt, ob sie mit wesentlichen Überzeugungen des gesellschaftlichen Bewusstseins im Einklang stehen oder nicht. Zweitens ist zu beachten, dass in politischer Praxis das Zusammenwirken dieser drei Dimensionen von Rationalität im Kontext stets potentiell neuartiger Handlungssituationen als Normalfall betrachten werden muss. Das schließt es nicht aus, wie der akteurzentrierte Institutionalismus einen Rationalitätsaspekt als für die Situationsdeutungen politischer Akteure typischerweise ausschlaggebend zu betrachten, solange man die jeweiligen Kontextbedingungen im Auge behält, unter denen diese Annahme gerechtfertigt ist. Davon wird aber nicht die diskurstheoretische Annahme berührt, dass eine politikwissenschaftliche Erklärung letztlich 
die Form einer rationalen Rekonstruktion annimmt. Stets geht es darum auszuloten, im welchem Verhältnis die verschiedenen Rationalitätsaspekte die Situationsdeutungen der Beteiligten im Einzelfall bestimmt haben, um auf dieser Basis die Bedeutung der vollzogenen Interaktion nachvollziehen zu können. Im Lichte dieser Erläuterungen zum Begriff rationaler Rekonstruktion lässt sich nun der soziologisch-explanative Geltungsanspruch nachzeichnen, der sich mit der Diskurstheorie des demokratischen Rechtsstaats verbindet.

\subsection{Rationale Rekonstruktion der Situation demokratischer Verfassungsgebung als Kern der Diskurstheorie des demokratischen Rechtsstaats}

Bekanntermaßen steht im Zentrum von Habermas' Theorie zum demokratischen Rechtsstaat ein Gedankenexperiment, dass bei oberflächlicher Betrachtung leicht mit dem Status der gedanklichen Konstruktion eines Natur- bzw. Urzustands in Theorien des Gesellschaftsvertrags verwechselt werden kann. Tatsächlich handelt es sich dabei aber dem Anspruch nach nicht um ein bloßes Darstellungsmittel einer philosophischen Konstruktion, sondern um den Vorschlag einer rationalen Rekonstruktion bestimmter historischer Interaktionssituationen: den Verfassungsgebungen von Paris und Philadelphia, mit denen die welthistorisch neue politische Praxis moderner Demokratie begründet worden ist (Habermas 2001). Habermas will erklären, warum die Organisationsform des demokratischen Rechtsstaats aus der Sicht der an diesen Ereignissen Beteiligten als rationale Antwort auf eine bestimmte Problemkonstellation erscheinen konnte. Im Zusammenhang von Habermas' Theorie verbinden sich mit dieser Rekonstruktion zwei empirische Hypothesen. Zum einen bildet sie das letzte Glied einer Theorie sozialer Evolution, die die Stufenfolge des historischen Weltbildwandels als Rationalisierungsprozess beschreibt. Zum anderen beansprucht Habermas (wie oben erläutert: auf der Basis dieser Theorie sozialer Evolution) den Geltungssinn nachzuvollziehen, der sich in den Ordnungen demokratischer Rechtsstaaten verkörpert und bis heute politische Praxis orientiert, weil sich an der Problemkonstellation der Ordnungsbildung, auf die der demokratische Rechtsstaat eine Antwort ist, in modernen westlichen Gesellschaften nichts grundlegend geändert hat. ${ }^{22}$

Der wesentliche Unterschied zwischen Habermas' Gedankenexperiment und philosophischen Urzustandskonstruktionen ist, dass jener dabei von den historischen Bedingungen ausgehen muss, die im zeitgenössischen Situationskontext vorlagen. Habermas nimmt an, dass die Situationsdeutung der Beteiligten von einer bestimmten politischen Konstellation sowie einem gewandelten moralischpraktischen und epistemischen Weltbild geprägt ist. Im Lichte weggebrochener sakraler Legitimationen der alten Herrschaftsordnung stellt sich den Beteiligten am Ende des 18. Jahrhunderts die Aufgabe, die politischen Machtverhältnisse auf eine neue Legitimationsgrundlage zu stellen, um die öffentliche Ordnung auf-

22 Habermas' jüngere Überlegungen zu einer mehrstufigen Weltordnung (Habermas 2004) sowie zur EU (Habermas 2011) diskutieren meines Erachtens vornehmlich, inwiefern sich die gleiche Problemkonstellation unter veränderten historisch-politischen Bedingungen zur Geltung bringt. Darauf kann ich an dieser Stelle aber nicht näher eingehen. 
rechtzuerhalten. Wie der akteurzentrierte Institutionalismus unterstellt Habermas für diesen politischen Kontext einen bestimmten Rationalitätsaspekt als zunächst für die Beteiligten ausschlaggebend: ein reflexiv gewordenes gesellschaftliches Bewusstsein. Im Lichte dieses Bewusstseins erscheint ihnen die Bewältigung des Problems der Neugründung einer politischen Ordnung, über „ihre“ jeweiligen kulturell-historischen Umstände hinaus, insbesondere an drei Bedingungen geknüpft. Erstens wissen die Beteiligten, dass zu den ordnungsbildenden Leistungen des Mediums des positiven, sanktionsbewehrten Rechts kein funktionales Äquivalent besteht. Angesichts sich als autonom und ungebunden begreifender Individuen ermöglicht allein staatlich sanktioniertes Recht eine effektive interaktionsübergreifende Regelung und kann so einerseits den Steuerungsbedarf komplex gewordener Gesellschaften decken sowie andererseits moralischen Normen gesellschaftsweit Nachachtung verschaffen. Zweitens lassen sich die Beteiligten von den beiden Gerechtigkeitsideen leiten, die die Grundpfeiler eines säkularisierten moralisch-praktischen Bewusstseins bilden: den Prinzipien der Selbstbestimmung und Selbstverwirklichung. Die zu etablierende politische Ordnung muss „im Einklang stehen mit den moralischen Grundsätzen einer bewusst entworfenen, selbstverantworteten Lebensführung von Einzelnen wie von Kollektiven“ (Habermas 1992, S. 128). Schließlich verfügen die Beteiligten über einen historischen (bzw. fallibilistischen) Sinn. Das heißt, sie wissen darum, dass sich eine heute als rational beurteilte Entscheidung im Lichte künftiger Entwicklungen als ungenügend herausstellen kann. Unter diesen Umständen macht es aus der Perspektive der Beteiligten Sinn, sich für die Ordnung des demokratischen Rechtsstaats zu entscheiden. Der demokratische Rechtsstaat ermöglicht die effektive, weil zwangsbewehrte rechtliche Regelung gesellschaftlicher Zusammenhänge; er realisiert über die demokratische Setzung des Rechts die Prinzipien der gleichberechtigten und gemeinschaftlichen Selbstbestimmung; und er institutionalisiert mit dem demokratischen Gesetzgebungsverfahren zugleich einen gesellschaftlichen Lernprozess, in welchem vormals getroffene Beschlüsse der Revision offenstehen (vgl. auch Peters 1996, S. 110-111). Im Dewey'schen Sinne könnte man sagen, der demokratische Rechtsstaat verkörpert die über Institutionen auf Dauer gestellte Rekonstruktion der Erfahrung einer politischen Gemeinschaft unter den Bedingungen der Gerechtigkeit.

Habermas argumentiert, die Verbindung von Recht, Staat und Demokratie im demokratischen Rechtsstaat, die westlichen Demokratien gemein ist, lässt sich als rationale Antwort auf das grundlegende Problem der Ordnungsbildung verstehen, wie es sich im Lichte eines modernen, reflexiv gewordenen gesellschaftlichen Bewusstseins darstellt. Die Ordnung des demokratischen Rechtsstaats unterwirft die kollektive Bearbeitung politischer Konflikte gleichermaßen den Anforderungen gemeinsamer moralischer, ethischer sowie epistemischer Reflexion - auch wenn sie die Spannungen, die sich im Einzelfall stets zwischen diesen Aspekten ergeben, nicht auflösen kann. 


\subsection{Ein Anwendungsbereich rationaler Rekonstruktion in empirischer Politikanalyse: Legitimationsforschung}

Abschließend soll die Analyse von Legitimationspraxis in der EU als ein möglicher Anwendungsbereich rationaler Rekonstruktion illustriert werden. Wie bereits erwähnt ist rationale Rekonstruktion in der Analyse politischer Praxis insbesondere dann nötig, wenn der institutionell geregelte Kontext politischen Handelns prekär ist. Unter diesen Umständen nimmt die Bedeutung von Rechtfertigungsprozessen als konstruktives Element von Politik entsprechend zu. Je schwächer die soziale Geltung einer politischen Ordnung desto stärker der Bedarf an Legitimationstätigkeit, die politisches Handeln, insbesondere von regierenden Akteuren, als im Einklang mit gesellschaftlich geteilten Werten und kognitiven Überzeugungen stehend sowie als Fortsetzung einer gemeinsamen Geschichte ausweist. Im Kontext der EU ist dieser Bedarf stärker ausgeprägt als im Rahmen nationalstaatlicher Politik. Die Dynamik der Herausbildung bzw. Transformation europäischer politischer Ordnung kann entsprechend auch an dem Ausmaß studiert werden, in dem sich in öffentlicher Legitimationspraxis politische Rechtfertigungsnarrative etablieren bzw. transformieren (Eder 2011; Forst u. Günther 2011), die eine legitimatorische Funktion erfüllen.

In der empirischen Legitimationsforschung lässt sich in jüngerer Zeit ein „communicative turn " (Schneider et al. 2007) feststellen, der sich auf diesen Umstand bezieht. Er wendet sich von der Tradition umfragegestützter Erfassung individueller Legitimitätsglauben (z. B. Fuchs u. Schneider 2011) unter der Annahme ab, dass Legitimität nicht als stabiler Zustand, sondern lediglich als Momentaufnahme fortwährender gesellschaftlicher Prozesse der Legitimation und Delegitimation $\mathrm{zu}$ verstehen ist. Entsprechend konzentriert sich diese Forschung auf die Analyse öffentlicher politischer Kommunikation. So werden einerseits öffentliche evaluative Sprechakte über einen bestimmten Zeitraum zahlenmäßig erfasst und unter Rechtfertigungstypen kategorisiert (Nullmeier et al. 2010). Andere Studien untersuchen politische Dokumente anhand theoretisch vorbestimmter Indikatoren, um die Verbreitung von „polity ideas“ (Diez 2001; Jachtenfuchs et al. 1998) oder Legitimationsnarrative in der europäischen Politik zu erfassen (Della Sala 2010; Wiener u. Hansen-Magnusson 2010).

Während diese Ansätze zu Recht die Aufmerksamkeit auf die Relevanz politischer Kommunikation lenken, bleiben sie durch ihren Fokus auf die Inhaltsebene von Legitimation meist unempfindlich für die spezifisch politische Dimension auf der pragmatischen Ebene von Rechtfertigungsprozessen. Anders die Literatur zu Framing-Prozessen in europäischer Politik (Daviter 2007; Rhinard 2010), welche den performativen Effekt politischer Kommunikation hervorhebt. Hier wird der gezielte Einsatz von Kommunikation als politisches Handeln begriffen, mit dem Akteure ihren Interessen im politischen Prozess Geltung verschaffen wollen. Als Framing bezeichnet man dabei den Versuch eines politischen Akteurs, mittels einer strategisch vorgebrachten Krisen- bzw. Problemdiagnose und darauf bezogener Lösungsvorschläge, die Erwirkung einer bestimmten Policy herbeizuführen (Rein u. Schön 1996). Im erfolgreichen Framing werden demnach Problemdiagnose und Lösungsvorschläge durch Bezug auf übergeordnet geltende normative 
Ordnungen in strategischer Weise als erforderlich und gerechtfertigt begründet, um künftige politische Auseinandersetzung über den Gegenstand im Sinne der je eigenen Interessen vorzustrukturieren.

Während Rechtfertigungsprozesse also einerseits in der Legitimationsforschung gelöst von Interessenpolitik betrachtet werden, kommen sie andererseits in der Framing-Analyse lediglich als Bestandteile einer (auch) kommunikationsgesteuerten Verfolgung des Eigeninteresses politischer Akteure in den Blick. Ein rekonstruktiver Ansatz muss dagegen davon ausgehen, dass beide Perspektiven in der hermeneutischen Analyse politischer Praxis zu integrieren sind. Es kommt dann darauf an, in der Erklärung politischer Praxis offen zu sein für die Erfassung dynamischer, kontextabhängiger Verhältnisse zwischen verschiedenen Rationalitätsaspekten. Das setzt unter anderem voraus, Legitimationspraxis als situationsgebundene Rechtfertigung im Zusammenhang politischer Interessenverfolgung zu verstehen. Anders formuliert, die strategische Formulierung einer Policy zielt zwar darauf, künftige Interaktion zu „framen“. Sie findet aber ebenso stets im Kontext eines normativen Hintergrunds geltender, übergeordneter Frames statt, der die Erfolgsbedingungen des strategischen Framings mitbestimmt. Da Herrschaftsakteure diese normative Konstellation im Zusammenhang von Interessenpolitik abwägen müssen, lässt sich Legitimationspraxis im Sinne einer Politik der Legitimation (Reus-Smit 2007, S. 159f.) begreifen. Im Kontext der sich stets transformierenden EU nimmt diese Politik der Legitimation insofern den Charakter einer Legitimationskonkurrenz an, als die Rechtfertigungspraxis eines Akteurs - wie zum Beispiel der EU-Kommission - neben der Rechtfertigung der EU als solcher und des je eigenen politischen Handelns stets auch auf das Rearrangement des institutionellen Gefüges der EU zu zielen scheint. Im Lichte eines solchen „Legitimationstrilemmas“ (Gaus u. Schmidtke 2012) bedarf die Analyse des Ausmaßes, in dem konkrete Rechtfertigungspraktiken europäischer politischer Akteure die strategische Verfolgung von Eigeninteressen, die Antizipation geltender gesellschaftlicher Hintergrundnormen und -überzeugungen bzw. institutionell vorgegebener Regeln oder gar eine Bedeutungsverschiebung derselben anzeigen, der rationalen Rekonstruktion im Einzelfall.

\section{Schluss}

Mit den vorangehenden Überlegungen zum Begriff der Rekonstruktion hoffe ich zweierlei erreicht zu haben. Zum einen sollte die bislang unterschätzte Bedeutung der Habermasschen Diskurstheorie für die politikwissenschaftliche Analyse hervorgehoben werden. Habermas' Begriff der Rekonstruktion ist nicht nur im Zusammenhang einer kritischen Gerechtigkeitstheorie und damit für den Kontext politischer Theorie in normativer Absicht relevant. Tritt man einen Schritt zurück und nimmt die logischen Verbindungslinien im Hintergrund des pluralistisch angelegten Werks in den Blick, zeigt sich die Relevanz seines Rekonstruktionsbegriffs auch im Kontext einer allgemeinen Theorie der Politik und ihrer Analysemethoden. Habermas' Programm einer rekonstruktiven Soziologie der Demokratie zeigt den Bedarf und das Ergänzungspotential hermeneutischer Verfahren zur empirischen Rekonstruktion politischer Praxis in der gegenwärtigen politik- 
wissenschaftlichen Methodenlandschaft an. Damit soll keine „Umdeutung“ der Diskurstheorie weg von normativer politischer Theorie hin zu einer empirisch orientierten Theorie der Politik vorgeschlagen werden. Das ließe sich angesichts der Ambivalenz, die in Habermas' Werk zwischen den Argumentationsmodi theoretischer Rekonstruktion und normativ-praktisch ausgerichteter philosophischer Konstruktion mitunter aufscheint (Peters 1996, S. 118-9), kaum rechtfertigen. Zu zeigen, dass dieses Changieren nicht als Ungenauigkeit, sondern als Ausdruck einer wissenschaftlichen Betrachtungsweise verstanden werden kann, die für die Funktion der Teildisziplin politische Theorie charakteristisch ist, ist das zweite Anliegen der vorgestellten Diskussion. Habermas führt einen zentralen Gedanken von Deweys Philosophie - das Bewusstsein welterschließender Praxis als fortlaufender Rekonstruktion menschlicher Erfahrung - insofern fort, als er ihn in seinen Konsequenzen sowohl für die normativ-kritische Betrachtung als auch für die empirisch verfahrende Analyse politischer Praxis weiterdenkt. In diesem Sinne kann Habermas' Theorie als ein Beispiel für die Brückenfunktion angesehen werden, die die Disziplin politische Theorie zwischen empirisch verfahrender Politikwissenschaft einerseits und Philosophie andererseits erfüllt. Ihre Vermittlungsleistung besteht darin, in verschiedenen Zusammenhängen „einen genuin philosophischen Gedanken wie einen Sprengsatz in eine spezielle Forschungssituation ein[zu] führ[en]“ (Habermas 1983a, S. 22).

\section{Literatur}

Aboulafia, Mitchell, Myra Bookman und Catherine Kemp (Hrsg.). 2002. Habermas and Pragmatism. London: Routledge.

Celikates, Robin. 2009. Kritik als soziale Praxis. Gesellschaftliche Selbstverständigung und kritische Theorie. Frankfurt a. M.: Campus.

Daviter, Falk. 2007. Policy Framing in the European Union. Journal of European Public Policy 14:654-666.

Della Sala, Vincent. 2010. Political Myth, Mythology and the European Union. Journal of Common Market Studies 48:1-19.

Dewey, John. 1920. Reconstruction in Philosophy. New York: Holt and Co.

Diez, Thomas. 2001. Europe as a Discursive Battleground: Discourse Analysis and European Integration Studies. Cooperation and Conflict 36:5-38.

Eder, Klaus. 2009. Communicative Action and the Narrative Structure of Social Life. The Social Embeddedness of Discourse and Market - a Theoretical Essay. In Critical Turns in Critical Theory: New Directions in Social and Political Thought, Hrsg. Séamas Ó'Tuama, 63-79. London: Tauris Academic Studies.

Eder, Klaus. 2011. Europe as a Narrative Network. Taking the Social Embeddedness of Identity Constructions Seriously. In Debating Political Identity and Legitimacy in the European Union, Hrsg. Sonia Lucarelli, Furi Cerutti und Vivien Schmidt, 38-54. London: Routledge.

Flügel, Oliver. 2008. Entzweiung. Die Normativität der Moderne. Baden-Baden: Nomos.

Forst, Rainer. 1999. Die Rechtfertigung der Gerechtigkeit. Rawls‘ Politischer Liberalismus und Habermas' Diskurstheorie in der Diskussion. In Das Recht der Republik, Hrsg. Hauke Brunkhorst und Peter Niesen, 105-168. Frankfurt a. M.: Suhrkamp. 
Forst, Rainer. 2007. Das Recht auf Rechtfertigung. Elemente einer konstruktivistischen Theorie der Gerechtigkeit. Frankfurt a. M.: Suhrkamp.

Forst, Rainer. 2011. The Justification of Justice: Rawls and Habermas in Dialogue. In Habermas and Rawls: Disputing the Political, Hrsg. James G. Finlayson und Fabian Freyenhagen, 153-180. New York: Routledge.

Forst, Rainer, und Klaus Günther. 2011. Die Herausbildung normativer Ordnungen. Zur Idee eines interdisziplinären Forschungsprogramms. In Die Herausbildung normativer Ordnungen: Interdisziplinäre Perspektiven, Hrsg. Rainer Forst und Klaus Günther, 1130. Frankfurt a. M.: Campus.

Fuchs, Dieter, und Christian Schneider. 2011. Support of the EU and European Identity: Some Descriptive Results. In Cultural Diversity, European Identity and the Legitimacy of the EU, Hrsg. Dieter Fuchs und Hans-Dieter Klingemann, 61-85. Northampton: Edward Elgar.

Fuchs-Goldschmidt, Inga. 2008. Konsens als normatives Grundprinzip der Demokratie. Zur Kritik der deliberativen Theorie der Demokratie. Wiesbaden: VS Verlag für Sozialwissenschaften.

Garz, Detlef. 2000. Kritik, Hermeneutik, Rekonstruktion. Über den Stellenwert der Methode bei Jürgen Habermas. In Das Interesse der Vernunft. Rückblicke auf das Werk von Jürgen Habermas seit „Erkenntnis und Interesse“, Hrsg. Stefan Müller-Doohm, 201217. Frankfurt a. M.: Suhrkamp.

Gaus, Daniel. 2009. Der Sinn von Demokratie. Die Diskurstheorie der Demokratie und die Debatte über die Legitimität der EU. Frankfurt a. M.: Campus.

Gaus, Daniel, und Oliver Schmidtke. 2013. Die EU-Kommission im Legitimationsdilemma. Eine Rekonstruktion der Legitimationspolitik im Weißbuch „Europäisches Regieren“. Sozialer Sinn (i.E.).

Geuss, Raymond. 2008. Philosophy and Real Politics, Princeton: Princeton University Press.

Gutmann, Amy, und Dennis Thompson. 2001. Deliberative Democracy. In Encyclopedia of Democratic Thought, Hrsg. Paul B. Clarke und Joe Foweraker, 137-141. New York: Routledge.

Habermas, Jürgen. 1973. Erkenntnis und Interesse. Frankfurt a. M.: Suhrkamp.

Habermas, Jürgen. 1981a. Theorie des kommunikativen Handelns. Bd. 1: Handlungsrationalität und gesellschaftliche Rationalisierung. Frankfurt a. M.: Suhrkamp.

Habermas, Jürgen. 1981b. Theorie des kommunikativen Handelns, Bd. 2: Zur Kritik der funktionalistischen Vernunft. Frankfurt a. M.: Suhrkamp.

Habermas, Jürgen. 1983a. Die Philosophie als Platzhalter und Interpret. In ders.: Moralbewußtsein und kommunikatives Handeln, 9-28. Frankfurt a. M.: Suhrkamp.

Habermas, Jürgen. 1983b. Rekonstruktive vs. verstehende Sozialwissenschaften. In ders.: Moralbewußtsein und kommunikatives Handeln, 29-52. Frankfurt a. M.: Suhrkamp.

Habermas, Jürgen. 1988. Handlungen, Sprechakte, sprachlich vermittelte Interaktionen und Lebenswelt. In ders.: Nachmetaphysisches Denken, 63-104. Frankfurt a. M.: Suhrkamp.

Habermas, Jürgen. 1992. Faktizität und Geltung. Beiträge zu einer Diskurstheorie des Rechts und des demokratischen Rechtsstaats. Frankfurt a. M.: Suhrkamp.

Habermas, Jürgen. 1999a. Realismus nach der sprachpragmatischen Wende. In ders.: Wabrheit und Rechtfertigung, 7-64. Frankfurt a. M.: Suhrkamp. 
Habermas, Jürgen. 1999b. Richtigkeit versus Wahrheit. Zum Sinn der Sollgeltung moralischer Urteile und Normen. In ders.: Wabrheit und Rechtfertigung, 271-318. Frankfurt a. M.: Suhrkamp.

Habermas, Jürgen. 2000. Nach dreißig Jahren: Bemerkungen zu „Erkenntnis und Interesse“. In Das Interesse der Vernunft. Rückblicke auf das Werk von Jürgen Habermas seit „Erkenntnis und Interesse“, Hrsg. Stefan Müller-Doohm, 12-20. Frankfurt a. M.: Suhrkamp.

Habermas, Jürgen. 2001. Der demokratische Rechtsstaat - eine paradoxe Verbindung widersprüchlicher Prinzipien? In ders.: Zeit der Übergänge, 133-151. Frankfurt a. M.: Suhrkamp.

Habermas, Jürgen. 2002. Postscript. Some Concluding Remarks. In Habermas and Pragmatism, Hrsg. Mitchell Aboulafia, Myra Bookman und Catherine Kemp, 223-233. London: Routledge.

Habermas, Jürgen. 2004. Hat die Konstitutionalisierung des Völkerrechts noch eine Chance? In ders.: Der gespaltene Westen, 113-193. Frankfurt a. M.: Suhrkamp.

Habermas, Jürgen. 2005. Kommunikatives Handeln und detranszendentalisierte Vernunft. In ders.: Zwischen Naturalismus und Religion, 27-83. Frankfurt a. M.: Suhrkamp.

Habermas, Jürgen. 2011. Zur Verfassung Europas. Ein Essay. Frankfurt a. M: Suhrkamp. Habermas, Jürgen. 2012. Rawls‘ Politischer Liberalismus. Replik auf die Wiederaufnahme einer Diskussion. In ders.: Nachmetaphysisches Denken II. Aufsätze und Repliken, 277-307. Frankfurt a. M.: Suhrkamp.

Honneth, Axel. 2007. Rekonstruktive Gesellschaftskritik unter genealogischem Vorbehalt. Zur Idee der „Kritik“ in der Frankfurter Schule. In ders.: Pathologien der Vernunft. Geschichte und Gegenwart der Kritischen Theorie, 57-69. Frankfurt a. M.: Suhrkamp. Honneth, Axel. 2011. Das Recht der Freiheit. Grundriß einer demokratischen Sittlichkeit. Frankfurt a. M.: Suhrkamp.

Iser, Mattias. 2008. Empörung und Fortschritt. Grundlagen einer kritischen Theorie der Gesellschaft. Frankfurt a. M.: Campus.

Jachtenfuchs, Markus, Thomas Diez und Sabine Jung. 1998. Which Europe? Conflicting Models of a Legitimate European Political Order. European Journal of International Relations 4:409-445.

Joas, Hans. 1992a. Die Kreativität des Handelns. Frankfurt a. M.: Suhrkamp.

Joas, Hans. 1992b. Die unglückliche Ehe von Hermeneutik und Funktionalismus. Über Jürgen Habermas‘ Theorie des kommunikativen Handelns. In ders.: Pragmatismus und Gesellschaftstheorie, 171-204. Frankfurt a. M.: Suhrkamp.

Jörke, Dirk. 2003. Demokratie als Erfahrung. John Dewey und die politische Philosophie der Gegenwart. Wiesbaden: Westdeutscher Verlag.

Landwehr, Claudia. 2009. Political Conflict and Political Preferences. Communicative Interaction Between Facts, Norms, and Interests. Colchester: ECPR Press.

Larmore, Charles. 2000. Habermas und der Pragmatismus. In Das Interesse der Vernunft. Rückblicke auf das Werk von Jürgen Habermas seit „Erkenntnis und Interesse“, Hrsg. Stefan Müller-Doohm, 328-343. Frankfurt a. M.: Suhrkamp.

MacIntyre, Alasdair. 1987. Der Verlust der Tugend. Zur moralischen Krise der Gegenwart. Frankfurt a. M./New York: Campus. 
Mayntz, Renate, und Fritz W. Scharpf. 1995. Der Ansatz des akteurzentrierten Institutionalismus. In Gesellschaftliche Selbstregelung und politische Steuerung, Hrsg. Renate Mayntz und Fritz W. Scharpf, 39-72. Frankfurt a. M./New York: Campus.

McCarthy, Thomas. 1994. Kantian Constructivism and Reconstructivism: Rawls and Habermas in Dialogue. Ethics 105:44-63.

Nullmeier, Frank, Dominika Biegon, Jennifer Gronau, Martin Nonhoff, Henning Schmidtke und Steffen Schneider. 2010. Prekäre Legitimitäten. Rechtfertigung von Herrschaft in der postnationalen Konstellation. Frankfurt a. M./New York: Campus.

Oevermann, Ulrich. 2000. Die Methode der Fallrekonstruktion in der Grundlagenforschung sowie der klinischen und der pädagogischen Praxis. In Die Fallrekonstruktion - Sinnverstehen in der sozialwissenschaftlichen Forschung, Hrsg. Klaus Kraimer, S. 58-156. Frankfurt a. M.: Suhrkamp.

Olsen, Johan P. 2010. Governing through Institution Building. Institutional Theory and Recent European Experiments in Democratic Organization, Oxford: Oxford University Press.

Pedersen, Jørgen. 2008. Habermas‘ Method: Rational Reconstruction. Philosophy of the Social Sciences 38:457-485.

Peters, Bernhard. 1996. On Reconstructive Legal and Political Theory. In Habermas, Modernity and Law, Hrsg. Mathieu Deflem, 101-134. London: Sage.

Rawls, John. 1975. Eine Theorie der Gerechtigkeit. Frankfurt a. M.: Suhrkamp.

Rehg, William, und James Bohman. 2002. Discourse and Democracy: The Formal and Informal Bases of Legitimacy in Between Facts and Norms. In Discourse and Democracy. Essays on Habermas's Between Facts and Norms, Hrsg. René von Schomberg und Kenneth Baynes, 31-60. Albany: State University of New York Press.

Rein, Martin, und Donald A. Schön. 1996. Frame-Critical Policy-Analysis and FrameReflective policy-Practice. Knowledge \& Society 9:85-104.

Reus-Smit, Christian. 2007. International Crisis of Legitimacy. International Politics 44:157-174.

Rhinard, Mark. 2010. Framing Europe: the Policy Shaping Strategies of the European Commission. Dordrecht: Republic of Letters.

Saar, Martin. 2009. Genealogische Kritik. In Was ist Kritik? Philosophische Positionen, Hrsg. Rahel Jaeggi und Tilo Wesche, 249-267. Frankfurt a. M.: Suhrkamp.

Scharpf, Fritz W. 2000. Interaktionsformen. Akteurzentrierter Institutionalismus in der Politikforschung. Wiesbaden: VS Velrag für Sozialwissenschaften.

Schaub, Jörg. 2010. Ideale und/oder nicht-ideale Theorie - oder weder noch? Ein Literaturbericht zum neuesten Methodenstreit in der politischen Philosophie. Zeitschrift für philosophische Forschung 64:393-409.

Schmalz-Bruns, Rainer. 1995. Reflexive Demokratie. Die demokratische Transformation moderner Politik. Baden-Baden: Nomos.

Schmalz-Bruns, Rainer, und Tanja Hitzel-Cassagnes. 2003. Normative/ökonomische politische Theorie. In Politikwissenschaft. Ein Grundkurs, Hrsg. Herfried Münkler, 132-171. Reinbek: rororo.

Schnädelbach, Herbert. 1992. Über Rationalität und Begründung. In ders.: Zur Rehabilitierung des animal rationale, 61-78. Frankfurt a. M.: Suhrkamp.

Schneider, Steffen, Frank Nullmeier und Achim Hurrelmann. 2007. Exploring the Communicative Dimension of Legitimacy: Text Analytical Approaches. In Legitimacy in an 
Age of Global Politics, Hrsg. Achim Hurrelmann, Steffen Schneider und Jens Steffek, 126-155. Houndmills: Palgrave.

Schweppenhäuser, Gerhard. 2005. Die Antinomie des Universalismus. Zum moralphilosophischen Diskurs der Moderne. Würzburg: Königshausen \& Neumann.

Simmons, A. John. 2010. Ideal and Nonideal Theory. Philosophy \& Public Affairs 38:5-36. Walzer, Michael. 1992. Sphären der Gerechtigkeit. Frankfurt a. M./New York: Campus.

Wiener, Antje, und Hannes Hansen-Magnusson. 2010. Studying Contemporary Constitutionalism: Memory, Myth and Horizon. Journal of Common Market Studies 48:21-44.

\section{Autorenangaben:}

Dr. Daniel Gaus,

Käte Hamburger Kolleg „Politische Kulturen der Weltgesellschaft”, Universität DuisburgEssen, Schifferstr. 196, 47059 Duisburg,

gaus@gcr21.uni-due.de 